\title{
Improving the BER Performance of M-FSK in a Noisy Multipath Rayleigh, and Rician Fading Channels Using Reed-Solomon Forward Error Correction Method
}

\author{
Bourdillon O. Omijeh ${ }^{1}$, Ekene Obodoekwe ${ }^{2}$ \\ ${ }^{1}$ Department of Electronic and Computer Engineering, University of Port Harcourt, Port Harcourt, Nigeria \\ ${ }^{2}$ Centre for Information and Telecommunication Engineering, University of Port Harcourt, Port Harcourt, Nigeria
}

Email address:

omijehb@yahoo.com (B. O. Omijeh), bourdillon.omijeh@uniport.edu.ng (E. Obodoekwe)

\section{To cite this article:}

Bourdillon O. Omijeh, Ekene Obodoekwe. Improving the BER Performance of M-FSK in a Noisy Multipath Rayleigh, and Rician Fading Channels Using Reed-Solomon Forward Error Correction Method. American Journal of Networks and Communications.

Vol. 5, No. 5, 2016, pp. 91-96. doi: 10.11648/j.ajnc.20160505.12

Received: August 22, 2016; Accepted: August 31, 2016; Published: September 24, 2016

\begin{abstract}
This paper focuses on the application of Reed-Solomon Error Correction Code evaluation technique on the BitError-Rate performance of M-ary Frequency Shift Keying (M-FSK) in noisy multipath Rayleigh and Rician fading channel. The detection and correction of the received information errors from the multipath fading channel was done using the Forward Error Correction (FEC) technique. The message is encoded and decoded using the error-correcting Reed-Solomon Codes were employed to encode and decode the message. The entire model based design, modelling and simulation was done using MATLAB/SIMULINK. It was observed that the BER performance of M-FSK with RS codes improved greatly compared to MFSK system without error correction. Decrease of BER performance was observed as the length of codeword symbols (n) increase at constant code rate and error correcting capability. The redundancy increases as code rate decreases leading to an improved Bit-Error-Rate performance. The improvement of the BER of M-FSK by using Reed-Solomon error correction code was achieved.
\end{abstract}

Keywords: MFSK, Reed-Solomon Code, Bit Error Rate, Multipath, Rayleigh, Rician

\section{Introduction}

Modern mobile and wireless communication systems adopt digital modulation scheme as an essential module for signal processing instead of previously used analog modulation. Digital modulation is very effective in dealing with noise and robust to channel impairments. The choice of which digital modulation scheme or technique to employ is largely dependent on cost and efficiency in providing high data rates and very low bit error rates (BER) in the midst of prevailing challenges such as fading [4, 5]. The major disadvantage is its high bandwidth requirement and poor BER performance when compared to other digital modulation techniques like BPSK and QPSK. Therefore, it is meaningful to adopt ways of improving the performance of a wireless mobile communication system with the deployment of M-ary (MFSK) modulation technique. The objective of this paper is to evaluate the BER performance of M-ary
Frequency Shift Keying (FSK) modulation scheme in a channel characterized by noise, Rayleigh fading (Non Line of Sight(LOS) fading), and Rician fading (LOS fading) using the Reed-Solomon Forward Error Correction method.

[1] In their paper, It was experimental that Binary input RS Encoder-Decoder provides an improved BER when compared to that of Integer input RS Encoder-Decoder, especially for the preferred smaller values of M. [2] In their paper, BER performance of M-FSK was tested under multichannel environments AWGN, Rayleigh fading and Rician fading channels with coherent and non- coherent demodulation and different sizes of modulation constellation. Convolutional code with hard and soft decision, extended Golay code and Reed-Solomon code, were used to improve the performance of the system under AWGN channel.

[3] In their paper, observed that the BER performance improves as the length of codeword symbols (n) increase at constant code rate Rc and error correcting capability increases. 


\section{Theoretical Background}

The FSK modulation for a signal can be expressed generally as,

$$
\begin{gathered}
S_{m}(t)=\sqrt{\frac{2 E}{T}} \operatorname{Cos}\left(2 \pi f_{m} t+\emptyset\right), 0 \leq t \geq T,\{m=1,2, \ldots, M\} \\
T R_{b}=\frac{K}{T} \text { bits } / \text { sec }
\end{gathered}
$$

and the corresponding information rate can be expressed as,

$$
R_{m}=R_{c} R_{b} \text { bits } / \mathrm{sec}
$$

where $R_{c}$ (code rate $=\frac{K}{N}$.

$$
\mathrm{R}_{\mathrm{b}}=\text { bit rate }
$$

\subsection{Reed-Solomon Codes}

Basically, Reed-Solomon [1] codes are non-binary systematic cyclic linear block codes. They are cyclic because each valid code produces another valid code when it is circularly shifted. They are linear because a new code word with the same length can be generated by adding any two valid code words. As the RS encoder processes each block of message symbols, represented as a sequence of m-bits with $\mathrm{m}$ as any positive integer which is greater than 2 , these codes are referred as Block codes.

The RS $(\mathrm{N}, \mathrm{K}, \mathrm{t})$ code parameters can be represented as follows [3].

Codeword symbols: $\mathrm{N}=2 \mathrm{~m}-1$

Information symbols: $\mathrm{k}=1,3, \ldots, \mathrm{n}-2$

Code minimum distance: $d_{\min }=\mathrm{n}-\mathrm{k}+1$ and

The error-correction capability symbols: $t=\frac{\left(d_{\min }-1\right)}{2}=$ $\frac{(n-k)}{2}$

\subsection{BER Mathematical Calculation of M-FSK System}

M- FSK with Coherent Detection in AWGN channel [5-8].

$$
\begin{gathered}
P_{s}=1-\int_{-\alpha}^{\alpha}\left[Q\left(-q-\sqrt{\frac{2 k E_{b}}{N_{o}}}\right)\right]^{M-1} \frac{1}{\sqrt{2 \pi}} \exp \left(-\frac{q^{2}}{2}\right) d q(4) \\
P_{b}=\frac{2^{k-1}}{2^{k}-1} P_{S}
\end{gathered}
$$

Where

$P_{s}$ : Symbol error rate (SER)

$P_{b}:$ Bit error rate (BER)

M: Size of modulation constellation

$\mathrm{K}:$ Number of bits per symbol $\rightarrow k=\log _{2} M$

$\frac{E_{b}}{N_{o}}$ : Energy per bit-to-noise power-spectral-density ratio.

- M-FSK with Non-coherent Detection in AWGN channel [5].

$$
\begin{gathered}
P_{s}=\sum_{m=1}^{M=1}(-1)^{m+1}\left(\begin{array}{c}
M-1 \\
m
\end{array}\right) \frac{1}{m+1} \exp \left[-\frac{m}{m+1} \frac{k E_{b}}{N_{o}}\right](6) \\
P_{b}=\frac{1}{2} \frac{M}{M-!} P_{s}
\end{gathered}
$$

$$
P_{M F S K}=1-\frac{1}{\sqrt{2 \pi}} \int_{-\alpha}^{\alpha} \exp \left[\left\{-\frac{1}{2}(y-\sqrt{2 \lambda})^{2}\right\}\right][1-Q(y)]^{M-1} d y
$$

MFSK shows in contrast to the other modulation schemes, the error probability decreases as $M$ increases.

- Reed-Solomon code with $N=Q-1=2^{q}[5]$

$$
P_{b}=\frac{1}{q} \frac{1}{N} \sum_{m=t+1}^{N} m\left(\begin{array}{l}
N \\
M
\end{array}\right)\left(P_{S}\right)^{m}\left(1-P_{S}\right)^{N-m}
$$

Otherwise,

$$
\text { If } \frac{\log _{2} Q}{\log _{2} M}=\frac{q}{k}=h
$$

Where $h$ is an integer

$$
P_{s}=1-(1-s)^{h}
$$

Where $s$ is the symbol error rate (SER) in uncoded AWGN channel.

- M-FSK in rayleigh fading channel [5]

$P_{S}=P_{b}=\frac{1}{2^{L}}\left(1-\sqrt{\frac{\bar{\gamma}}{2+\bar{\gamma}}}\right)^{L} \sum_{k=0}^{L-1}\left(\begin{array}{c}L-1+k \\ k\end{array}\right) \frac{1}{2^{k}}\left(1+\sqrt{\frac{\bar{\gamma}}{2+\bar{\gamma}}}\right)^{k}$

Where

L: diversity branch

$M_{\gamma l}$ Moment generating functions for each diversity branch

For Rayleigh fading:

$$
M_{\gamma l}(s)=\frac{1}{1-s \overline{\gamma l}}
$$

- M-FSK in rician fading channel [5]

$$
\begin{aligned}
& P_{S}=\sum_{r}^{M-1} \frac{(-1)^{r+1} e^{-\frac{L K \bar{\gamma}_{r}}{1+\gamma_{r}}}}{\left(r\left(1+\bar{\gamma}_{r}\right)+1\right)^{L}}\left(\begin{array}{c}
M-1 \\
r
\end{array}\right)^{r} \sum_{n=0}^{L-1} \frac{\Gamma(L+n)}{\Gamma(L)}\left[\frac{1+\bar{\gamma}_{r}}{r+1+r \bar{\gamma}_{r}}\right]^{n} \\
& P_{b}=\frac{1}{2} \frac{M}{M-1} P_{S} \\
& \bar{\gamma}_{r}=\frac{1}{1+K} \bar{\gamma} \\
& \beta_{n r}=\sum_{i=n-(L-1)}^{n} \frac{\beta_{i(r-1)}}{(n-i) !} I_{[0,(r-1)(L-1)]}(i) \\
& \beta_{00}=\beta_{r 0}=1 \\
& \beta_{n 1}=\frac{1}{n !} \\
& \beta_{1 r}=r
\end{aligned}
$$

And $I_{[a, b]}(i)=1$ ifa $\leq i \geq$ band 0 otherwise.

Where $\mathrm{K}$ is the ratio of energy in the specular component to the energy in the diffuse component (linear scale).

For identically-distributed diversity branches:

$$
M_{\gamma l}(s)=M_{\gamma} \text { for all } l \text {. }
$$




\section{Model-Based Design Steps}

The model used in this paper was designed with Simulink [9-11].

- Select Random integer Generator block from the Channels sub library of the Communications.

- Select Communications system toolbox $\rightarrow$ Error Detection and correction $\rightarrow$ Block $\rightarrow$ Integer-input RS encoder, and decoder blocks

- Select DSP system Toolbox $\rightarrow$ Signal Management $\rightarrow$ Buffers $\rightarrow$ Buffer, and Unbuffer blocks.

- Select Communications toolbox $\rightarrow$ Modulation $\rightarrow$ Digital

Baseband Modulation $\rightarrow$ FM $\rightarrow$ M-FSK Modulator Baseband and Demodulator Baseband blocks. Fig 4: Configuration parameters for M-FSK Modulator and Demodulator blocks

- Select Communications system toolbox $\rightarrow$ Modulation $\rightarrow$ Interleaving $\rightarrow$ Block $\rightarrow$ Matrix Interleaver and Deinterleaver blocks.

- Select Communications system toolbox $\rightarrow$ Comm Sinks $\rightarrow$ Error Rate Calculation Block.

- Select Sinks Sub library $\rightarrow$ Display Block

- Select Commonly Used Blocks sub library $\rightarrow$ Gain block.

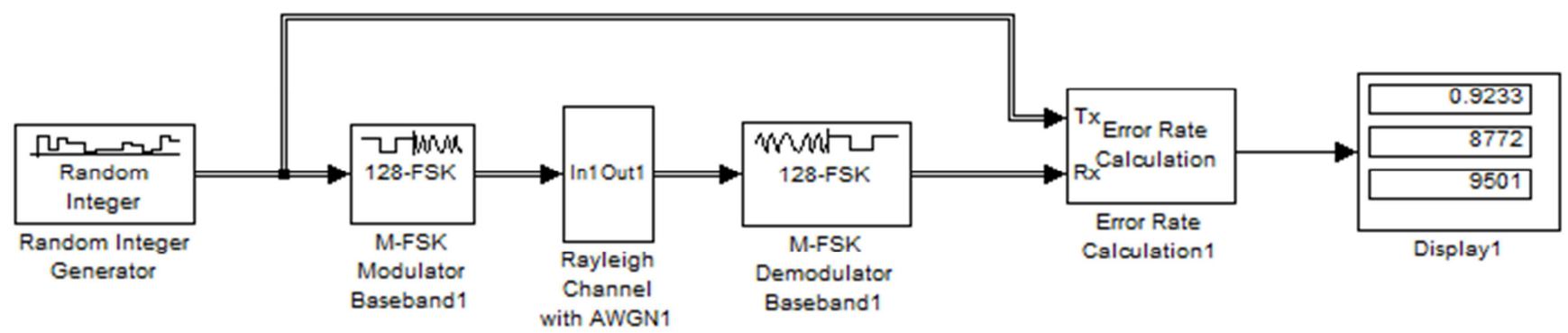

Fig. 1. Simulink Model of M-FSK system in Multipath Rayleigh Fading Channel without RS code.

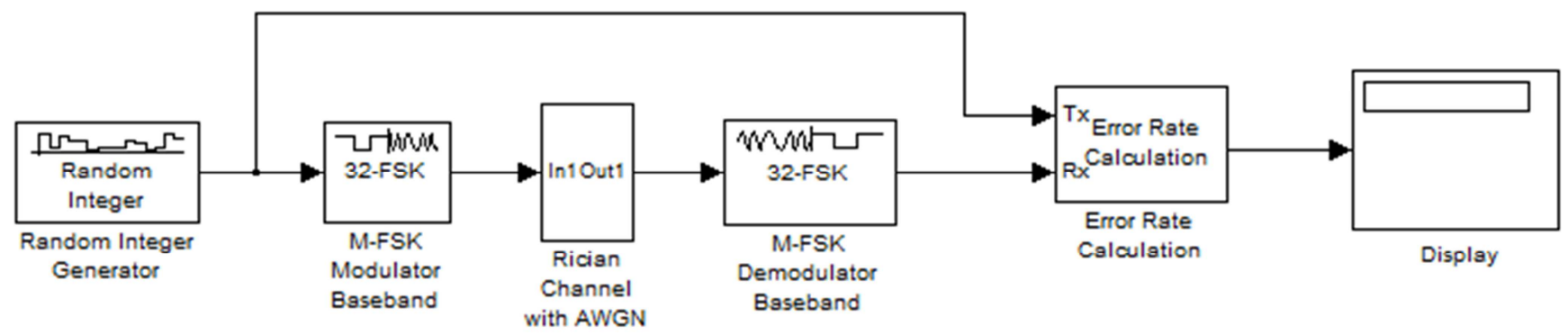

Fig. 2. Simulink Model of M-FSK system in Rician Fading Channel without RS code.

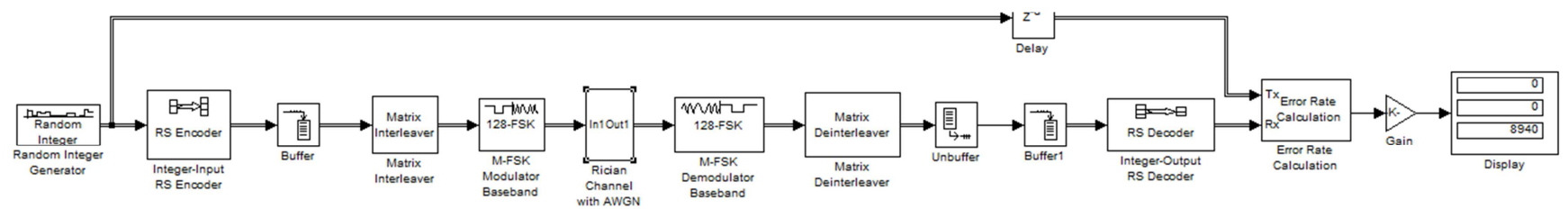

Fig. 3. Simulink Model of M-FSK in Multipath Rician Fading channel with AWGN.

The Simulink model for the Rayleigh and Rician channels with AWGN are shown in Fig. 4 below.

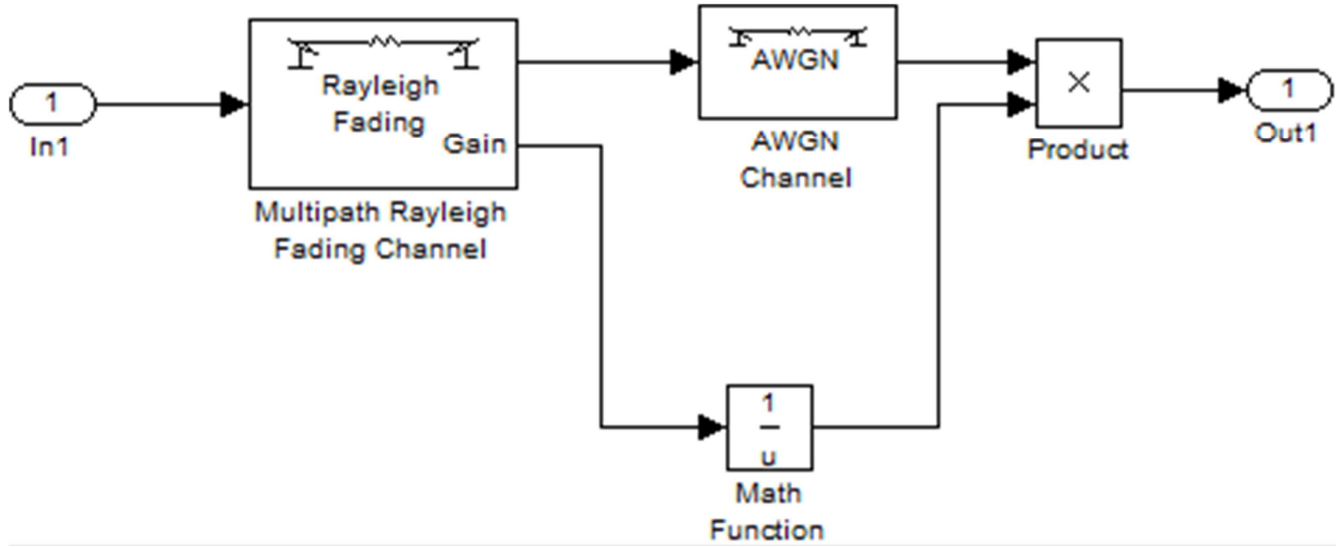




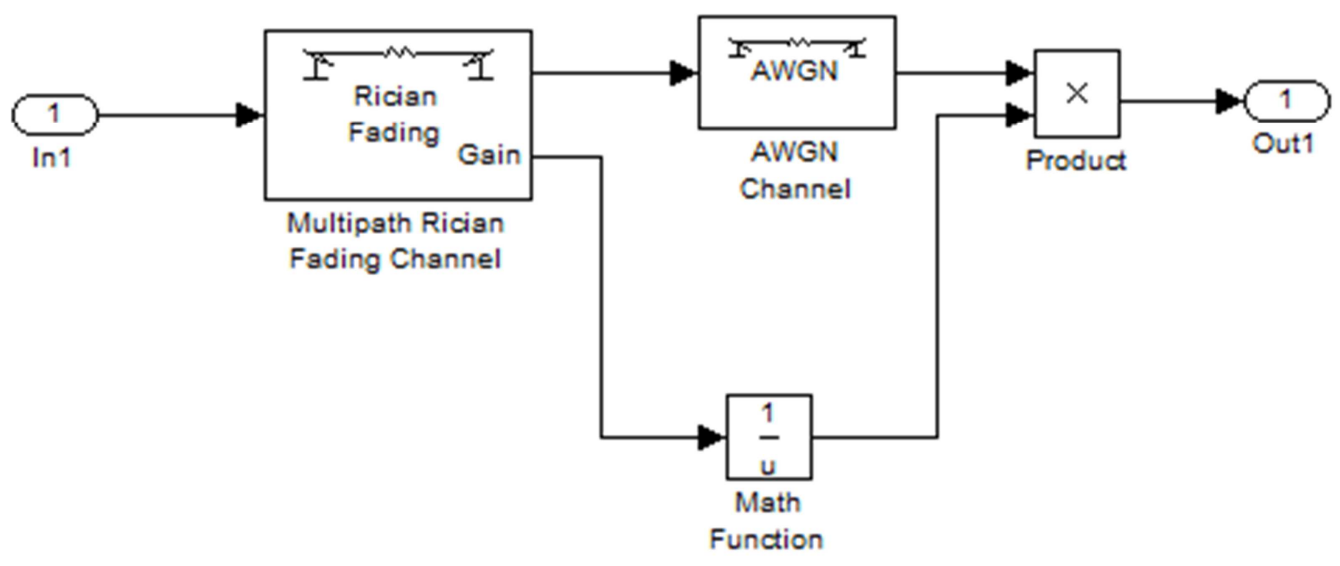

Fig. 4. Simulink model for the Multipath Rayleigh and Rician channels with AWGN.

\section{Simulation Results and Discussion}

Simulation time: 25000 Matlab seconds

Table 1. BER performance of Uncoded M-FSK system.

\begin{tabular}{llll}
\hline MFSK UNCODED & & & \\
\hline $\mathbf{E s}=\mathbf{E b} / \mathbf{N o}+\mathbf{E b} / \mathbf{N o} * \log 10(\mathbf{M})$ & & & $\mathbf{2 0 d b}$ \\
\hline $\mathbf{5 d b}$ & $\mathbf{1 0 d b}$ & $\mathbf{1 5 d b}$ & 0.6757 \\
\hline 0.7813 & 0.6757 & 0.6757 & 0.8197 \\
0.8400 & 0.8403 & 0.8197 & 0 \\
0.9434 & 0.9009 & 0.9009 & \\
\hline
\end{tabular}

Table 2. BER performance for Multi Rayleigh channel with RS code $(n, k, t)$.

\begin{tabular}{llllll}
\hline \multicolumn{1}{l}{ Rayleigh Channel } & & & & \\
\hline $\mathbf{E s} / \mathbf{N o}=\mathbf{E b} / \mathbf{N o}+\mathbf{E b} / \mathbf{N o} * \log \mathbf{1 0}(\mathbf{M} * \mathbf{k} / \mathbf{n})$ & & & $\mathbf{1 5 d b}$ & $\mathbf{2 0 d b}$ \\
\hline $\mathbf{M}$ & $\mathbf{C o d e}-w o r d$ & $\mathbf{E b} / \mathbf{N o}=\mathbf{5 d b}$ & $\mathbf{1 0 d b}$ & 0 & 0 \\
\hline 32 & $(31,15)$ & 0.2531 & 0.006262 & 0 & 0 \\
64 & $(63,47)$ & 0.1364 & 0.008187 & 0 & 0 \\
128 & $(127,111)$ & 0.06072 & 0.01192 & 0 \\
\hline
\end{tabular}

Table 3. BER performance for Rayleigh channel with different code-word.

\begin{tabular}{|c|c|c|c|c|c|}
\hline \multicolumn{6}{|c|}{ Rayleigh Channel } \\
\hline \multicolumn{6}{|c|}{$E s / N o=E b / N o+E b / N o * \log 10(M * k / n)$} \\
\hline M & Code-word & $\mathrm{Eb} / \mathbf{N o}=5 \mathrm{db}$ & $10 \mathrm{db}$ & $15 \mathrm{db}$ & 20db \\
\hline 32 & $(31,19)$ & 0.1645 & 0.005749 & 0 & 0 \\
\hline 64 & $(63,51)$ & 0.04814 & 0.008525 & 0 & 0 \\
\hline 128 & $(127,115)$ & 0.04108 & 0.007121 & $8.987 \mathrm{e}^{\wedge}-05$ & 0 \\
\hline
\end{tabular}

Table 4. BER performance for Rayleigh channel with different code-word

\begin{tabular}{lllllll}
\hline $\mathbf{M}$ & Code-word & $\mathbf{E b} / \mathbf{N o}=\mathbf{5 d b}$ & $\mathbf{1 0 d b}$ & $\mathbf{1 5 d b}$ & $\mathbf{2 0 d b}$ & $\mathbf{C o d e} \mathbf{r a t e}=\mathbf{k} / \mathbf{n}$ \\
\hline 32 & $(31,15)$ & 0.2531 & 0.006262 & 0 & 0 & 0.4839 \\
64 & $(63,31)$ & 0.2762 & 0.0007498 & 0 & 0 & 0.4762 \\
128 & $(127,61)$ & 0.2897 & 0 & 0 & 0 & 0.4803 \\
16 & $(15,7)$ & 0.2159 & 0.009917 & $6.428 \mathrm{e}^{\wedge}-5$ & 0 & 0.4920 \\
\hline
\end{tabular}

Table 5. Multipath Rician channel (no code-word).

\begin{tabular}{|c|c|c|c|c|}
\hline \multicolumn{5}{|c|}{ Multipath Rician channel (no code word) } \\
\hline \multicolumn{5}{|c|}{$\mathrm{Es}=\mathrm{Eb} / \mathrm{No}+\mathrm{Eb} / \mathrm{No} * \log 10(\mathrm{M})$} \\
\hline $\mathbf{M}$ & $E b / N_{0}=5 d b$ & $10 \mathrm{db}$ & $15 \mathrm{db}$ & $20 \mathrm{db}$ \\
\hline 16 & 0.9317 & 0.9375 & 0.9434 & 0.9434 \\
\hline 32 & 0.9677 & 0.9804 & 0.9868 & 0.9868 \\
\hline 64 & 0.9804 & 0.9804 & 0.9804 & 0.9804 \\
\hline
\end{tabular}


Table 6. Multipath Rician channel (code-word).

\begin{tabular}{lllllll}
\hline \multicolumn{2}{l}{ Multipath Rician channel } & & & & \\
\hline M & Code word & $\mathbf{5 d b}$ & $\mathbf{1 0 d b}$ & $\mathbf{1 5 d b}$ & $\mathbf{2 0 d b}$ & Code rate=k/n \\
\hline 32 & $(31,15)$ & 0.1931 & $4.209 \mathrm{e}^{\wedge}-05$ & 0 & 0 & 0.4839 \\
16 & $(15,7)$ & 0.1587 & $8.57 \mathrm{e}^{\wedge}-05$ & 0 & 0 & 0.4927 \\
64 & $(63,31)$ & 0.26221 & 0 & 0 & 0 & 0.4762 \\
\hline
\end{tabular}

Table 7. Multipath Rician channel with different Code Rate(CR).

\begin{tabular}{|c|c|c|c|c|c|c|}
\hline \multicolumn{7}{|l|}{ M-ary number } \\
\hline & 32 & & 64 & & 128 & \\
\hline \multicolumn{7}{|l|}{ BIT ERROR RATE } \\
\hline \multirow{2}{*}{$\begin{array}{l}\mathrm{Es} / \mathrm{No}=\mathrm{Eb} / \mathrm{No}+\mathrm{Eb} / \mathrm{No}^{*} \log 10 \\
(\mathrm{M} * \mathrm{k} / \mathbf{n})\end{array}$} & $(31,15,8)$ & $(31,19,6)$ & $(63,47,8)$ & $(63,51,6)$ & $(127,111,6)$ & $(127,115,8)$ \\
\hline & $\mathrm{CR}=0.48$ & 0.61 & 0.75 & 0.81 & 0.87 & 0.91 \\
\hline $5 \mathrm{db}$ & 0.2531 & 0.1645 & 0.1364 & 0.08814 & 0.06072 & 0.04108 \\
\hline $10 \mathrm{db}$ & 0.006262 & 0.005749 & 0.008187 & 0.008525 & 0.01192 & 0.007121 \\
\hline $15 \mathrm{db}$ & 0 & 0 & 0 & 0 & 0 & $8.987 \mathrm{e}^{\wedge}-05$ \\
\hline $20 \mathrm{db}$ & 0 & 0 & 0 & 0 & 0 & 0 \\
\hline
\end{tabular}

Case 1: R-S FEC M-FSK system vs Uncoded M-FSK system

Table 1 shows the BER performance of M-FSK without forward error correction. Table 2 shows the BER performance of M-FSK with Reed-Solomon Forward Error Correction method under multipath Rayleigh Fading channel (the nature of the channel is shown in Fig 4). Case 1 compares BER recorded in table 1 against the BER recorded in table 2 under the same channel. Case 1 also compares the BER performance of M-FSK with FEC under multipath Rician fading channel against the BER performance of $\mathrm{M}$ FSK without FEC. The results show that the BER greatly improved for different values of different values of Energy per symbol to Noise power spectral density (Es/No) because of the Reed-Solomon Error correction method. The poor BER performance of M-FSK without error correction is greatly improved when used with Reed-Solomon forward error correction method. It is observed from the simulation results that the BER performance of the M-FSK system with Reed-Solomon FEC is much better under multipath Rician fading channel than under multipath Rayleigh fading channel.

Case 2: behaviour as the Function of Code length

The results showed in Table 4 and Table 6 show the performance of M-FSK as a function of code length at constant code rate. As shown in Tables 3 and 4, the value of the code rate is held nearly constant (at 0.48 ). It is observed that the BER improves as the codeword symbols (N) decreases from 127 to 16 for different values of Energy per symbol to Noise power spectral density (Es/No).It is observed from the simulation results that at constant code rate, the BER performance of the M-FSK system with ReedSolomon FEC is much better under multipath Rician fading channel than under multipath Rayleigh fading channel.

Case 3: Performance as the Function of Redundancy

In the results shown in Table 7 with RS coding, the RS code sets used have identical codeword symbols $(\mathrm{N})$ whereas the number of information symbols $(\mathrm{K})$ decreases, that apparently resulted in different error correction capability $(\mathrm{t})$
6 and 8respectively. Also, the code rate $(\mathrm{Rc}=\mathrm{k} / \mathrm{n})$ is increased from 0.48 to 0.91 . It is observed that the BER performance increases as the error correction capability ( $\mathrm{t}$ ) decreases from 8 to 6 at a constant codeword symbol $(\mathrm{N})$.

\section{Conclusion}

In this paper, the improvement of Bit-Error-Rate (BER) performance of M-FSK in a noisy multipath Rayleigh and Rician fading channel using Reed-Solomon forward error correction method has been achieved. BER improvement can be obtained if RS coding is properly chosen. The following deductions are made:

i. BER performance of the M-FSK system with ReedSolomon FEC is much better under multipath Rician fading channel than under multipath Rayleigh fading channel

ii. At constant code rate, the BER performance of the MFSK system with Reed-Solomon FEC is much better under multipath Rician fading channel than under multipath Rayleigh

iii. The BER performance increases as the error correction capability (t) decreases from 8 to 6 at a constant codeword symbol $(\mathrm{N})$ (meaning an increase in the code rate of the RS code from 0.48 to 0.91 ).

\section{References}

[1] T. A. Shanmugasundaram, Dr. Alamelu Nachaiappan (2013): "An Insight into BER Performance of Reed Solomon Coded M-FSK under AWGN, Rayleigh and Rician Fading Channels", International journal of advance research in electrical, electronic and instrumentation engineering, vol. 2, issue 4, April 2013.

[2] Fatima Faydhe Al-azzawi, Saleim Hachemfarhan, Maher Ibraheemgamej (2013): "M-FSK in Multi Coding and Channel Environment", International journal of soft computing \& engineering (IJSCE) ISSSN: 2231-2307, Vol. 5, issue 3, july 2015 . 
[3] Saurabhmahajan, Gurpadamsingh (2011): "BER performance of Reed Solomon code using M-ary FSK modulation in AWGN channel", International journal of advance in science \& technology, Vol. 3, No.1, 2011.

[4] O. P. Sharma, V. Janyani and S. Sancheti (2008): "Analysis of Bit Error Rate Improvement in MFSK Communication Link", World Academy of Science, Engineering and Technology International Journal of Electrical, Computer, Energetic, Electronic and Communication Engineering Vol. 2, No. 9, 2008.

[5] F. F. Al-Azzawi, S. H. Farhan, M. I. Gamaj (2015): "M-FSK in Multi Coding and Channel Environments", International Journal of Soft Computing and Engineering (IJSCE) ISSN: 2231-2307, Volume-5 Issue-3, July 2015.

[6] G. Tharakanatha1, SK. Mahaboob kamal basha2, Vijay Bhaskar chanda3, I. Hemalatha 4 (2013): Implementation and Bit Error Rate analysis of BPSK Modulation and Demodulation Technique using MATLAB, International Journal of Engineering Trends and Technology (IJETT) Volume 4 Issue 9- Sep 2013.pp: 4010-4014.
[7] B. O. Omijeh and S. D. O. Agoye (2015): Comparative Evaluation of Different Digital Modulation Schemes on AWGN, LOS and Non-LOS Fading Channels Based on BER Performance. International Journal of Scientific \& Engineering Research, Volume 6, Issue 10, October-2015.

[8] Ruchi G, Arora. A. K, Akhilesh k. Y: Comparison of Different Multipath Channel, Based on Bit ErrorRate by Using Higher Order Modulation Techniques For Wcdma, International Journal of Advances in Engineering Science and Technology, www.sestindia.org/volume-ijaest/ and www.ijaestonline.com, pp:73-81, accessesd 2016

[9] Weizheng. W (1997): Simulink Communication Toolbox, Mathworks, Inc. http:www.mathwork.com

[10] Omijeh. B. O (2016): Applied Engineering Mathematics with Matlab/Simulink, M \& J Grand Orbit Communications Ltd, Port Harcourt, 2016; PP: 201-203. 